\title{
Renalase is associated with adverse left atrial remodelling and disease burden in patients with atrial fibrillation undergoing pulmonary vein isolation
}

\author{
Maciej T. Wybraniec ${ }^{1,2 *}$, Joanna Wieczorek ${ }^{1 *}$, Iwona Woźniak-Skowerska ${ }^{1}$, Andrzej Hoffmann ${ }^{1}$, \\ Seweryn Nowak ${ }^{1}$, Małgorzata Cichoń ${ }^{1}$, Krzysztof Szydło ${ }^{1}$, Anna Wnuk-Wojnar ${ }^{1}$, Jerzy Chudek ${ }^{3}$, \\ Andrzej Więcek ${ }^{4}$, Katarzyna Mizia-Stec ${ }^{1}$
}

'First Department of Cardiology, School of Medicine in Katowice, Medical University of Silesia, Katowice, Poland 2Upper Silesia Medical Centre, Katowice, Poland

${ }^{3}$ Department of Pathophysiology, School of Medicine in Katowice, Medical University of Silesia, Katowice, Poland ${ }^{4}$ Department of Nephrology, Transplantation, and Internal Medicine, School of Medicine in Katowice, Medical University of Silesia, Katowice, Poland

*Both authors contributed equally to this work.

\begin{abstract}
Background: Renalase is a catecholamine-metabolising enzyme, but its possible association with atrial fibrillation (AF) remains unknown.

Aim: We sought to evaluate plasma renalase concentration in patients with AF undergoing pulmonary vein isolation (PVI) with respect to AF clinical characteristics, left atrial (LA) remodelling, and PVI efficacy.

Methods: This case-control study included 69 patients (median age 58 years) with either paroxysmal (89\%) or persistent (11\%) AF, referred for PVI, and a control group consisting of 15 patients without AF, matched for age, sex, and comorbidities. An evaluation of transthoracic echocardiography with LA speckle tracking and plasma renalase concentration using an enzyme-linked immunosorbent assay was performed. AF recurrence was defined as any AF episode on seven-day electrocardiographic monitoring at six-month follow-up.

Results: Renalase level was higher in the study group than in the control group (mean $27.99 \mathrm{vs} .21 .48 \mu \mathrm{g} / \mathrm{mL}, \mathrm{p}=0.004$ ), but it was lower in patients with persistent AF (19.05 vs. $28.77 \mu \mathrm{g} / \mathrm{mL} ; \mathrm{p}=0.023)$ and among patients with AF episodes directly preceding PVI (24.50 vs. $29.66 \mu \mathrm{g} / \mathrm{mL} ; \mathrm{p}=0.04)$. Renalase concentration within the first quartile was associated with higher mean heart rate (70 vs. $61 \mathrm{bpm}, \mathrm{p}=0.029)$, greater AF burden (36.9\% vs. 9.3\%, $p=0.027$ ), greater LA diameter (41.1 vs. $37.9 \mathrm{~mm}, \mathrm{p}=0.011)$, and a trend towards less negative global LA strain $(-9.4 \mathrm{vs} .-13.5, p=0.082)$. Logistic regression revealed that global four-chamber LA strain was the only independent predictor of renalase variability $(p=0.0045)$. Renalase concentration did not predict AF recurrence at six-month follow-up (area under curve $[A U C]=0.614, p=0.216$ ). Conclusions: Low renalase level may be associated with impaired rate control, higher AF burden, and advanced LA remodelling in AF patients undergoing PVI, but it does not predict sinus rhythm maintenance.
\end{abstract}

Key words: renalase, atrial fibrillation, pulmonary vein isolation, speckle tracking

Kardiol Pol 2018; 76, 8: 1232-1241

\section{Address for correspondence:}

Maciej T. Wybraniec, MD, PhD, First Department of Cardiology, School of Medicine in Katowice, Medical University of Silesia, ul. Ziołowa 47, 40-635 Katowice, Poland, tel: +48 3235988 90, e-mail: maciejwybraniec@gmail.com; wybraniec@os.pl

Received: 15.02.2018 Accepted: 23.04.2018 Available as AoP: 25.04.2018

Kardiologia Polska Copyright (C) Polish Cardiac Society 2018 


\section{INTRODUCTION}

Atrial fibrillation (AF) remains one of the leading challenges of contemporary medicine due to its widespread prevalence and ischaemic stroke risk [1]. None of the hitherto published studies has unequivocally proven the superiority of either rhythm or rate control strategy in terms of mortality reduction [2, 3]; however, in patients with new-onset AF, especially among young adults, preservation of sinus rhythm is frequently preferred on account of the prevention of electrical left atrial (LA) remodelling [4]. Rhythm control strategy incorporates antiarrhythmic drugs, pharmacological or electrical cardioversion, and pulmonary vein isolation (PVI). Despite rapid improvement of technology and accessibility, PVI leads to objective improvement (absence of $\mathrm{AF}$ or reduction of $\mathrm{AF}$ episodes duration and intensity) in just $60 \%$ of patients $[5,6]$. Due to possible procedural complications and high cost, the cornerstone of successful PVI relies on adequate qualification and selection of procedural responders.

Several predictors of AF recurrence have been established, such as the number of comorbidities, AF episode duration, $\mathrm{CHADS}_{2}$ and $\mathrm{CHA}_{2} \mathrm{DS}_{2}$-VASc scores $[7,8]$, LA diameter [9] or volume [10], LA fibrosis as assessed by late gadolinium enhancement on cardiac magnetic resonance [11, 12], LA global longitudinal strain [13], or LA appendage emptying flow velocity [14]. Also, several biomarkers served as promising predictors of adverse LA remodelling and sinus rhythm maintenance, including natriuretic peptides $[15,16]$, C-reactive protein and interleukin-6 [17], galectin-3 [18], endothelin-1 [16, 19], and cardiac troponin [10, 16]. However, none of the listed biomarkers alone conferred sufficient diagnostic power to predict the outcome of AF treatment.

Renalase represents a circulating protein, expressed primarily in proximal tubular cells and the myocardium [20, 21]. Although its interference with metabolism of catecholamines is disputed, renalase has been shown to inversely correlate with peripheral and central blood pressure [22]. Single nucleotide polymorphisms within the renalase gene were documented to be related with primary arterial hypertension [23], ventricular hypertrophy, and left ventricular dysfunction [24]. Exogenous renalase protected myocardial cells against ischaemia-reperfusion injury in an animal model [25]. We therefore speculated that renalase may play a role in LA atrial remodelling and thereby potentially modulate response to PVI and facilitate sinus rhythm maintenance.

Thus, the purpose of the study was to evaluate plasma renalase concentration in patients with paroxysmal or persistent $\mathrm{AF}$ undergoing $\mathrm{PVI}$ with regard to clinical characteristics of $\mathrm{AF}$ and echocardiographic indices of adverse left atrial remodelling and recurrence.

\section{METHODS}

The project was conducted as a case-control study. The study flow-chart is depicted in Figure 1. Sixty-nine patients with either paroxysmal or persistent AF undergoing catheter-based percutaneous PVI during index hospitalisation were enrolled into the study group, which was furthered categorised into four subgroups depending on the quartile of peripheral vein renalase distribution. The control group consisted of $15 \mathrm{vol}-$ unteer patients consulted in the cardiology clinic, without current or former $\mathrm{AF}$, as verified by 24-h electrocardiographic monitoring, matched for sex, age, and comorbidities with the study group. In both groups different clinical and echocardiographic parameters, as well as plasma renalase concentration were evaluated.

Patients in the study group were followed up for six months on an outpatient basis. At this time point, a seven-day electrocardiographic monitoring was performed to detect possible recurrence of $\mathrm{AF}$ and to establish six-month follow-up $\mathrm{AF}$ burden. The primary endpoint was any form of AF detected on Holter monitoring at six months.

Patients in both the study and control groups were diligently queried and previous discharge summaries were analysed to elucidate the following exclusion criteria: a) history of transient ischaemic attack or stroke of any kind; b) active neoplastic disease; c) inflammatory disease within the past three months; d) chronic kidney disease with estimated glomerular filtration rate $<30 \mathrm{~mL} / \mathrm{min} / 1.73 \mathrm{~m}^{2}$; e) liver dysfunction (any hepatic aminotransferase $>3 \times$ upper reference limit); f) valvular heart disease (moderate or severe); g) left ventricular ejection fraction $<45 \%$, congestive heart failure; and $\mathrm{h}$ ) idiopathic cardiomyopathy.

The study was conducted in accordance with the Declaration of Helsinki and was approved by the Ethics Committee of the Medical University of Silesia in Katowice. All the patients gave written consent for participation in the study prior to enrolment.

\section{Laboratory tests and renalase concentration}

Blood samples $(10 \mathrm{~mL})$ were collected: a) from the antecubital vein, on admission, in all study participants (study and control groups); b) from the femoral vein, at the beginning of the PVI procedure, in the study group.

Blood samples were assayed for plasma renalase concentration by means of enzyme-linked immunosorbent assay (ELISA) method (Cloud-Clone Corp., Houston, TX, USA). EDTA was used as an anticoagulant, and samples were centrifuged for $15 \mathrm{~min}$ at $1000 \times \mathrm{g}$ at $2^{\circ} \mathrm{C}$ to $8^{\circ} \mathrm{C}$ within $15 \mathrm{~min}$ of collection. The samples were stored at $-80^{\circ} \mathrm{C}$ with no freeze-thaw cycles and assayed within three months following acquisition according to the manufacturer's instructions.

\section{Electrocardiographic Holter monitoring}

Prolonged 24-h electrocardiographic monitoring was performed during index hospitalisation prior to PVI in the study group and on an outpatient basis in the control group. At six months the patients in the study group underwent a seven-day 


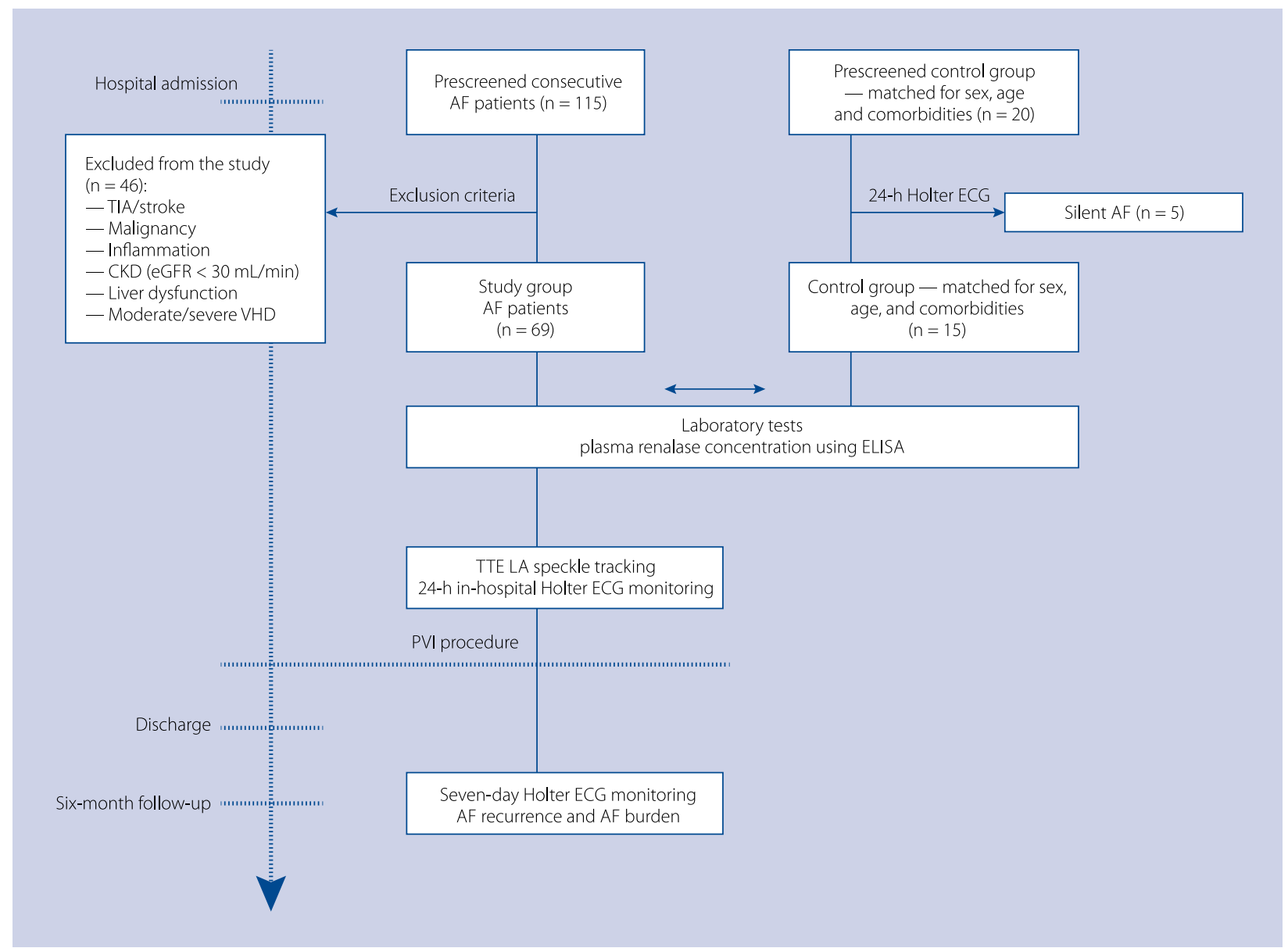

Figure 1. Study flow-chart including the study and control groups; AF — atrial fibrillation; CKD — chronic kidney disease; ECG - electrocardiography; eGFR — estimated glomerular filtration rate; ELISA — enzyme-linked immunosorbent assay; $\mathrm{PVI}$ - pulmonary vein isolation; TIA — transient ischaemic attack; TTE — transthoracic echocardiography; VHD — valvular heart disease

Holter electrocardiographic monitoring to assess individual response to PVI. AF burden was defined as the percentage of overall duration of $\mathrm{AF}$ episodes in relation to the total time of recording. The monitoring was performed using the Pathfinder SL system (Spacelabs Healthcare, Deerfield, WI, USA).

\section{Transthoracic echocardiography and speckle tracking imaging}

Routine echocardiographic check-up was conducted using Epiq 7G (Philips, Andover, MA, USA) with a 2.5-MHz probe in two-dimensional (2D), $M-$, and Doppler modes by a single investigator. Offline 2D speckle tracking imaging was employed to calculate two-chamber and four-chamber LA global longitudinal strains using EchoPAC Software version 112.0.0 (GE Healthcare, Horten, Norway). Subsequently, carotid scans were performed using a Logic 7, GE ultrasound machine with a 7- to 12-MHz linear array transducer and then analysed using a quantitative analysis package manufactured by Siemens. The scans were acquired perpendicularly to the carotid artery, and measurements of intima-media thickness were made at 1 -mm intervals over a 10-mm segment of the vessel. The maximal and mean intima-media thickness measurements were determined for the proximal and distal walls of the left and right common carotid arteries.

\section{Definitions}

Paroxysmal AF was defined as self-terminating within $48 \mathrm{~h}$ or cardioverted within seven days from the onset of arrhythmia, while persistent AF was defined as arrhythmia lasting for more than seven days [1]. Arterial hypertension was diagnosed if blood pressure was above the threshold of $140 / 90 \mathrm{mmHg}$ on two separate measurements during index hospitalisation or in the event of former arterial hypertension diagnosis or antihypertensive medication use. Diabetes mellitus/impaired fasting glucose/impaired glucose tolerance were analysed jointly. Diabetes was diagnosed on the condition that fasting blood exceeded $125 \mathrm{mg} / \mathrm{dL}$ on two separate days or if a patient received insulin or oral hypoglycaemic agents. Estimated glomerular filtration rate was calculated according to Cockroft-Gault formula. 


\section{Periprocedural management}

All patients were anticoagulated with vitamin $\mathrm{K}$ antagonists for at least three months prior to PVI. Transoesophageal echocardiography was performed to exclude the potential presence of LA appendage thrombus within $24 \mathrm{~h}$ preceding the procedure. Three or four venous accesses were obtained by Seldinger technique: for the left (a 6-F sheath - quadripolar electrode placed in the right ventricle) and right femoral veins (two 8-F sheaths - circular mapping and radiofrequency ablation electrodes or one 14-F sheath for cryoablation balloon) and for the right jugular vein (a 6-F sheath - decapolar electrode for coronary sinus catheterisation). Rotational angiography of LA was performed at the beginning of the procedure (contrast medium injection to pulmonary artery during fast right ventricular stimulation). Following transseptal puncture, three-dimensional electro-anatomical mapping was performed using the CARTO $^{\circledR} 3$ system (Biosense Webster, Diamond Bar, CA, USA) in patients undergoing radiofrequency ablation. PVI was achieved by means of either radiofrequency ablation with the ThermoCool ${ }^{\circledR}$ SmartTouch ${ }^{\circledR}$ SF catheter (Biosense Webster, Diamond Bar, CA, USA) ( $n=61,88.4 \%)$ or with balloon cryoablation ( $\mathrm{n}=8,11.6 \%$ ) using an Arctic Front Advance $^{\mathrm{TM}}$ catheter (Medtronic, Minneapolis, MN, USA). PVI was performed under the guidance of a circular mapping electrode - Lasso (Biosense Webster, Diamond Bar, CA, USA) or Achieve (Medtronic, Minneapolis, MN, USA). The procedure was performed with international normalised ratio (INR) up to 2.5 (preferably 2.0-2.5). All the patients received an IV bolus of unfractioned heparin $(100 \mathrm{IU} / \mathrm{kg})$ immediately after transseptal puncture and continuous infusion of $2000 \mathrm{IU} / \mathrm{h}$ through a transseptal sheath to obtain an activated clotting time (ACT) of over $300 \mathrm{~s}$. The ACT was measured at 30-min intervals during the procedure. Postprocedural 24-h heparin infusion under the control of ACT was continued in patients with INR $<2$ during the procedure. The next vitamin $\mathrm{K}$ antagonist dose was administered $4 \mathrm{~h}$ after the procedure to obtain an INR within the range of 2.0-3.0 the following day.

\section{Statistical analysis}

Statistical analysis was performed using Statistica 10.0 (StatSoft, Krakow, Poland) software. Quantitative variables were presented as the mean \pm standard deviation (SD) or median (interquartile range), and qualitative parameters were expressed as numbers and percentages. The type of distribution was verified using Shapiro-Wilk test. In case of normally-distributed variables, Student $t$ test for unpaired samples was used, while the Mann-Whitney $U$ test was implemented in non-normally distributed parameters. The association between initial renalase concentration and $\mathrm{AF}$ recurrence was established using receiver operating characteristic curve (ROC) analysis. Multiple regression analysis covered all the variables with $p<0.1$ in the univariate regression model. A p-value $<0.05$ was regarded as statistically significant.

\section{RESULTS \\ General characteristics}

The demographic and clinical characteristics of the study group are shown in Table 1. In brief, the study group consisted of 69 patients with either paroxysmal (89.9\%) or persistent AF (10.1\%), who were referred for catheter-based radiofrequency PVI. The majority of patients were men $(66.7 \%)$ and the median age was 58 years. The study group was characterised by high symptomatology of AF (median European Heart Rhythm Association [EHRA] class 3) and low ischaemic stroke risk (median $\mathrm{CHA}_{2} \mathrm{DS}_{2}$-VASc score of one point). All of the study participants received vitamin $\mathrm{K}$ antagonist as their anticoagulation regimen. In the course of in-hospital stay, one patient exhibited moderate access site haematoma without a decrease in haemoglobin concentration.

\section{Study versus control group}

The intergroup comparison is presented in Table 2. Patients in both groups were characterised by comparable rates of comorbidities, such as arterial hypertension and diabetes. Patients in the study group had lower white blood cell count $(p=0.006)$ and platelet count $(p=0.056)$. Plasma renalase concentration was markedly higher in the study group than in the control group ( $27.99 \mathrm{vs.} 21.48 \mu \mathrm{g} / \mathrm{mL}, \mathrm{p}=0.004)$.

\section{Renalase in the study group}

In the study group, subjects with paroxysmal AF had markedly higher levels of peripheral vein renalase $(28.77 \pm$ \pm 9.48 vs. $19.05 \pm 12.60 \mu \mathrm{g} / \mathrm{mL}, \mathrm{p}=0.048$ ) in comparison to patients with persistent $\mathrm{AF}$ (Table 3).

Plasma renalase levels did not differ depending on the presence or absence of arterial hypertension $(p=0.431$ and $p=0.229$, respectively), type 2 diabetes mellitus $(p=0.893$ and $p=0.381)$, coronary artery disease $(p=0.375$ and $p=0.152)$, or obesity $(p=0.282$ and $p=0.117)$. However, cigarette smokers exhibited lower levels of plasma renalase concentration $(20.05 \pm 12.02$ vs. $28.50 \pm 9.00 \mu \mathrm{g} / \mathrm{mL}, \mathrm{p}=0.026)$.

Patients with in-hospital episodes of AF during the acquisition of samples directly preceding PVI had lower renalase level (24.496 vs. $29.66 \mu \mathrm{g} / \mathrm{mL} ; \mathrm{p}=0.04)$ and simultaneously had a greater LA volume index (31.5 vs. $25.0 \mathrm{~mL} / \mathrm{m}^{2}$, $p=0.041)$ and less negative global four-chamber LA strain $(-10.9$ vs. $-14.2, p=0.03)$.

\section{Atrial fibrillation severity and plasma renalase concentration}

The distribution of different clinical variables within four quartiles of plasma renalase concentration in the study group is denoted in Table 4 and Figure 2. In comparison to the fourth quartile, patients within the lowest quartile of renalase concentration had greater mean and maximal heart rate on preprocedural electrocardiographic monitoring, greater $\mathrm{AF}$ burden and LA diameter, and less negative global longitudinal 
Table 1. Demographic, clinical, and procedural characteristics of the study group

\begin{tabular}{|c|c|c|}
\hline \multicolumn{2}{|l|}{ Variable } & Value \\
\hline \multicolumn{2}{|l|}{ Male sex } & $46(66.7)$ \\
\hline \multicolumn{2}{|l|}{ Age [years] } & $58(50 ; 62)$ \\
\hline \multicolumn{2}{|c|}{ Body mass index $\left[\mathrm{kg} / \mathrm{m}^{2}\right]$} & $28.7 \pm 4.4$ \\
\hline \multicolumn{2}{|c|}{$\mathrm{CHA}_{2} \mathrm{DS}_{2}$-VASc [points] } & $1(1 ; 2)$ \\
\hline \multicolumn{2}{|c|}{ EHRA score } & $3(2 ; 3)$ \\
\hline \multicolumn{2}{|l|}{ Persistent AF } & $7(10.1)$ \\
\hline \multicolumn{2}{|l|}{ History of PVI } & $2(2.9)$ \\
\hline \multicolumn{2}{|l|}{ History of CV } & $17(24.6)$ \\
\hline \multirow[t]{2}{*}{ Mode of PVI: } & RF & $61(88.4)$ \\
\hline & Cryoablation & $8(11.6)$ \\
\hline \multirow[t]{3}{*}{ AF duration: } & $0-5$ years & $29(42.0)$ \\
\hline & $5-10$ years & $24(34.8)$ \\
\hline & $>10$ years & $16(23.2)$ \\
\hline \multicolumn{2}{|c|}{ Cigarette smoking } & $28(40.6)$ \\
\hline \multicolumn{2}{|c|}{ Obesity } & $22(31.9)$ \\
\hline \multicolumn{2}{|c|}{ Coronary artery disease } & $14(20.3)$ \\
\hline \multicolumn{2}{|c|}{ Arterial hypertension } & $41(59.4)$ \\
\hline \multicolumn{2}{|c|}{ Hyperlipidaemia } & $45(65.2)$ \\
\hline \multicolumn{2}{|c|}{ Type 2 diabetes } & $12(17.4)$ \\
\hline \multicolumn{2}{|c|}{ Thyroid disease } & $19(27.5)$ \\
\hline \multicolumn{2}{|c|}{ ACEI } & $36(52.2)$ \\
\hline \multicolumn{2}{|l|}{$\beta$-blockers } & $49(71.0)$ \\
\hline \multicolumn{2}{|c|}{ Vitamin $\mathrm{K}$ antagonists } & $69(100)$ \\
\hline \multicolumn{2}{|c|}{ Amiodarone } & $6(8.7)$ \\
\hline Propafenone & & $31(44.9)$ \\
\hline Sotalol & & $9(13.0)$ \\
\hline Statins & & $31(44.9)$ \\
\hline Duration of in & spital Holter monitoring [h] & $19.0(17.8 ; 19.3)$ \\
\hline Maximal HR [b & & $100(83 ; 115)$ \\
\hline Mean HR [bpn & & $66(57 ; 75)$ \\
\hline Minimal HR [b & & $52(46 ; 55)$ \\
\hline AF burden [\%] & & $22.6 \pm 38.7$ \\
\hline Maximal HR d & g $\mathrm{AF}[\mathrm{bpm}]$ & $134.5(112.0 ; 147.0)$ \\
\hline Patients with i & ospital AF episodes & $20(28.9)$ \\
\hline LA volume ind & {$\left[\mathrm{mL} / \mathrm{m}^{2}\right]$} & $25.6 \pm 9.4$ \\
\hline LVEF [\%] & & $58.6 \pm 4.4$ \\
\hline Global four-ch & ber LA LS & $-11.5 \pm 4.7$ \\
\hline Global two-ch & ber LA LS & $-12.0 \pm 4.9$ \\
\hline$E / E^{\prime}$ & & $8.2 \pm 2.9$ \\
\hline Carotid intima & edia thickness [mm] & $0.65(0.57 ; 0.86)$ \\
\hline Platelet count & $\left.1000 / \mathrm{mm}^{3}\right]$ & $196.7 \pm 46.3$ \\
\hline White blood c & count $\left[\times 1000 / \mathrm{mm}^{3}\right]$ & $5.9 \pm 1.3$ \\
\hline Haemoglobin & centration $[\mathrm{g} / \mathrm{dL}]$ & $14.2 \pm 1.3$ \\
\hline Thyroid-stimul & hg hormone $[\mu \mathrm{lU} / \mathrm{mL}]$ & $2.3(0.99 ; 3.1)$ \\
\hline Total cholester & {$[\mathrm{mg} / \mathrm{dL}]$} & $183.29 \pm 44.55$ \\
\hline Serum creatini & concentration $[\mathrm{mg} / \mathrm{dL}]$ & $0.91 \pm 0.15$ \\
\hline eGFR $[\mathrm{mL} / \mathrm{min} /$ & $\left.73 \mathrm{~m}^{2}\right]$ & $82.91 \pm 8.31$ \\
\hline Six-month ECC & olter monitor duration [h] & $166.5(144.4 ; 168.5)$ \\
\hline Six-month foll & -up — AF burden [\%] & $7.1 \pm 20.7$ \\
\hline Six-month follo & up - patients with AF episodes & $12(17.4)$ \\
\hline Plasma renalas & oncentration $[\mu \mathrm{g} / \mathrm{mL}]$ & $27.99 \pm 10.01$ \\
\hline
\end{tabular}

Data are shown as absolute count and percentage, or median and interquartile range, or mean \pm standard deviation. ACEI - angiotensin converting enzyme inhibitor; $A F$ - atrial fibrillation; $C V$ - electrical cardioversion; ECG - electrocardiographic; E/E' — the ratio of mitral peak velocity of early filling (E) to early diastolic mitral annular velocity (E'); eGFR - estimated glomerular filtration rate; EHRA — European Heart Rhythm Association; HR — heart rate; LA — left atrial; LS — longitudinal strain; LVEF — left ventricular ejection fraction; PVI — pulmonary vein isolation; RF — radiofrequency
LA strain (Table 4, Fig. 2). The rest of the echocardiographic indices of LA remodelling, and LA and left ventricular systolic and diastolic functions were comparable between the first and fourth quartile of plasma renalase concentration.

After exclusion of patients with persistent AF, patients with plasma renalase concentration within the first quartile were characterised by a trend towards higher mean heart rate $(69.5[57 ; 82] \mathrm{bpm}$ vs. 61.5 [57; 66] bpm, $\mathrm{p}=0.072$ ), less negative four-chamber global LA strain $(-9.7 \pm 4.6$ vs. $-13.4 \pm 5.7, p=0.11)$, and a trend towards greater LA diameter $(40.3 \pm 2.2 \mathrm{~mm}$ vs. $38.2 \pm 3.5 \mathrm{~mm}$, $p=0.087$ ) in comparison with the highest levels of plasma renalase (fourth quartile).

\section{Renalase and $A F$ recurrence at six-month follow-up}

At six-month follow-up, episodes of AF were documented in $12(17.4 \%)$ patients who underwent the initial PVI procedure. Patients with evidence of AF on seven-day continuous electrocardiogram monitoring at six-month follow-up were characterised by comparable levels of plasma renalase concentration $(p=0.38)$. The ROC curve analysis showed that plasma renalase concentration (area under curve $[\mathrm{AUC}]=0.614$, 95\% confidence interval $[\mathrm{Cl}] 0.473-0.743, p=0.216$; Fig. 3 ) did not have sufficient power for prediction of AF recurrence.

\section{Univariate and multivariate regression analyses}

Linear regression analysis demonstrated that plasma renalase concentration negatively correlated with mean heart rate, maximal heart rate during AF episode and AF burden, LA diameter, and global longitudinal four-chamber strain (Table 5). Conversely, renalase level positively corresponded with mean platelet volume (Table 5).

According to stepwise multivariate regression analysis, global longitudinal four-chamber LA strain was the only independent predictor of plasma renalase concentration $(b=-0.425, p=0.0045$; adjusted coefficient of determination $\mathrm{R}^{2}=0.18$ ).

\section{DISCUSSION}

The current research shed light on the association between plasma renalase level and the markers of structural and electrical LA remodelling and $\mathrm{AF}$ burden. It was documented that patients with evidence of AF have substantially higher plasma renalase concentration (Table 2). Although plasma renalase did not accurately predict AF recurrence at six months after percutaneous $\mathrm{PVI}$, patients with plasma renalase within the lowest quartile were characterised by higher AF burden during index hospitalisation, greater LA diameter, and less negative LA global longitudinal strain (Fig. 2, Table 4). Moreover, patients with paroxysmal AF had higher levels of plasma renalase concentration than the subgroup with persistent $\mathrm{AF}$, while current AF episode corresponded with lower levels of plasma renalase. 
Table 2. Comparison of study and control groups in terms of different clinical variables and peripheral vein renalase concentration

\begin{tabular}{|c|c|c|c|}
\hline Variable & $\begin{array}{c}\text { Study group } \\
\text { — patients with AF } \\
(n=69)\end{array}$ & $\begin{array}{c}\text { Control group } \\
\text { — patients without AF } \\
\qquad(n=15)\end{array}$ & p \\
\hline Male sex & $46(66.7)$ & $9(60.0)$ & $0.416^{\mathrm{a}}$ \\
\hline Age [years] & $58(50 ; 62)$ & $57(53 ; 58)$ & $0.900^{b}$ \\
\hline Body mass index $\left[\mathrm{kg} / \mathrm{m}^{2}\right]$ & $28.7 \pm 4.4$ & $31.0 \pm 4.2$ & $0.426^{c}$ \\
\hline Arterial hypertension & $41(59.4)$ & $10(66.7)$ & $0.415^{\mathrm{a}}$ \\
\hline Type 2 diabetes & $12(17.4)$ & $3(20.0)$ & $0.531^{\text {a }}$ \\
\hline Platelet count $\left[\times 1000 / \mathrm{mm}^{3}\right]$ & $196.7 \pm 46.3$ & $220.9 \pm 30.2$ & $0.056^{b}$ \\
\hline White blood cells [×1000/mm³] & $5.9 \pm 1.3$ & $7.0 \pm 2.1$ & $0.006^{c}$ \\
\hline Haemoglobin concentration $[\mathrm{g} / \mathrm{dL}]$ & $14.2 \pm 1.3$ & $14.5 \pm 1.4$ & $0.387^{c}$ \\
\hline Serum creatinine concentration [mg/dL] & $0.91 \pm 0.15$ & $0.88 \pm 0.2$ & $0.770^{c}$ \\
\hline $\mathrm{eGFR}\left[\mathrm{mL} / \mathrm{min} / 1.73 \mathrm{~m}^{2}\right]$ & $82.9 \pm 8.3$ & $90.7 \pm 16.9$ & $0.390^{c}$ \\
\hline Plasma renalase concentration $[\mu \mathrm{g} / \mathrm{mL}]$ & $27.99 \pm 10.01$ & $21.48 \pm 5.01$ & $0.004^{b}$ \\
\hline
\end{tabular}

Data are shown as number (percentage), median and interquartile range, or mean \pm standard deviation. Conversion factors to SI units are as follows: for creatinine [in $\mathrm{mg} / \mathrm{dL}$ ] -88.4 ; for haemoglobin [in $\mathrm{g} / \mathrm{dL}]-10$; for total cholesterol [in $\mathrm{mg} / \mathrm{dL}$ ] -0.02586 . Abbreviations - see Table 1 aFisher's exact test; ${ }^{b}$ Mann-Whitney U test; 'Student's t-test

Table 3. Comparison of patients with paroxysmal and persistent atrial fibrillation in the study group

\begin{tabular}{|c|c|c|c|}
\hline Variable & Paroxysmal AF $(n=62)$ & Persistent AF $(n=7)$ & p \\
\hline Male sex & $41(89.1)$ & $5(71.4)$ & 0.361 \\
\hline Age [years] & $59(50 ; 62)$ & $46(39 ; 57)$ & 0.092 \\
\hline Body mass index $\left[\mathrm{kg} / \mathrm{m}^{2}\right]$ & $28.9 \pm 4.5$ & $27.7 \pm 2.8$ & 0.528 \\
\hline Arterial hypertension & $37(59.7)$ & $4(57.1)$ & 0.551 \\
\hline Type 2 diabetes & $12(19.4)$ & $1(14.3)$ & 0.296 \\
\hline Coronary artery disease & $13(21.0)$ & $2(16.7)$ & 0.640 \\
\hline eGFR $\left[\mathrm{mL} / \mathrm{min} / 1.73 \mathrm{~m}^{2}\right]$ & $82.6 \pm 8.4$ & $84.8 \pm 8.0$ & 0.544 \\
\hline Haemoglobin concentration [g/dL] & $14.1 \pm 1.2$ & $14.9 \pm 2.1$ & 0.143 \\
\hline Platelet count $\left[\times 1000 / \mathrm{mm}^{3}\right]$ & $193.8 \pm 44.7$ & $211.3 \pm 51.2$ & 0.370 \\
\hline $\mathrm{CHA}_{2} \mathrm{DS}_{2}$-VASc [points] & $1(1 ; 2)$ & $1(1 ; 2)$ & 0.646 \\
\hline EHRA score & $3(2 ; 3)$ & $2(2 ; 2)$ & 0.002 \\
\hline Maximal HR [bpm] & $100(83.5 ; 113)$ & $147.5(113 ; 153.5)$ & 0.028 \\
\hline Mean HR [bpm] & $66(57 ; 73)$ & $75.5(65.5 ; 81.5)$ & 0.364 \\
\hline Minimal HR [bpm] & $51.5(45.5 ; 55.0)$ & $55.0(51.5 ; 59.0)$ & 0.459 \\
\hline LA diameter $[\mathrm{mm}]$ & $39.0 \pm 3.9$ & $41.4 \pm 5.2$ & 0.210 \\
\hline LA volume index $\left[\mathrm{mL} / \mathrm{m}^{2}\right]$ & $25.1 \pm 9.4$ & $27.9 \pm 5.5$ & 0.608 \\
\hline LVEF [\%] & $58.6 \pm 4.3$ & $58.0 \pm 5.7$ & 0.769 \\
\hline Global four-chamber LA LS & $-11.8 \pm 4.8$ & $-6.9 \pm 2.3$ & 0.075 \\
\hline Global two-chamber LA LS & $-12.3 \pm 4.8$ & $-6.3 \pm 0.5$ & 0.018 \\
\hline$E / E^{\prime}$ & $8.2 \pm 3.1$ & $7.7 \pm 2.3$ & 0.768 \\
\hline Plasma renalase concentration $[\mu \mathrm{g} / \mathrm{mL}]$ & $28.77 \pm 9.48$ & $19.05 \pm 12.60$ & 0.048 \\
\hline
\end{tabular}

Data are shown as number (percentage), median and interquartile range or mean \pm standard deviation. Abbreviations - see Table 1

Our study is the first report in the literature regarding renalase concentration in the population of patients with $A F$, thus it cannot be directly compared to any former studies. The present findings comply with previous reports suggesting the role of renalase in promoting cardiomyocyte survival [25] via PMCA4b receptor and mitogen-activated protein 
Table 4. Distribution of various clinical parameters depending on the quartile of peripheral vein renalase concentration

\begin{tabular}{|c|c|c|c|c|c|c|}
\hline \multirow[t]{2}{*}{ Variable } & \multicolumn{4}{|c|}{ Plasma renalase concentration } & \multirow[t]{2}{*}{$p^{*}$} & \multirow[t]{2}{*}{$p^{* *}$} \\
\hline & $1^{\text {st }}$ quartile & $2^{\text {nd }}$ quartile & $3^{\text {rd }}$ quartile & $4^{\text {th }}$ quartile & & \\
\hline Age [years] & $59(50 ; 65)$ & $56(42 ; 58)$ & $59(55 ; 62)$ & $61(52 ; 63)$ & $0.716^{b}$ & $0.191^{b}$ \\
\hline Body mass index $\left[\mathrm{kg} / \mathrm{m}^{2}\right]$ & $29.2 \pm 4.0$ & $30.1 \pm 4.2$ & $26.5 \pm 3.8$ & $29.0 \pm 5.2$ & $0.792^{a}$ & $0.062^{a}$ \\
\hline $\mathrm{CHA}_{2} \mathrm{DS}_{2}$-VASc [points] & $1.5(1 ; 3)$ & $1.0(1 ; 2)$ & $1.0(1 ; 2)$ & $1.0(1 ; 2)$ & $1.0^{\mathrm{b}}$ & $0.588^{b}$ \\
\hline EHRA score & $2(2 ; 3)$ & $2(2 ; 3)$ & $3(3 ; 3)$ & $3(2 ; 3)$ & $0.114^{b}$ & $0.038^{b}$ \\
\hline Maximal HR [bpm] & $120.5(86.5 ; 153.0)$ & $107.5(88 ; 114)$ & $93.5(77 ; 112)$ & $91(86 ; 101)$ & $0.037^{b}$ & $0.158^{b}$ \\
\hline Mean HR [bpm] & $70.0(57.0 ; 81.5)$ & $70.5(62 ; 76)$ & $62.5(56 ; 70)$ & $61.0(57 ; 66)$ & $0.029^{b}$ & $0.133^{b}$ \\
\hline Minimal HR [bpm] & $53(47 ; 55)$ & $53.5(48 ; 56)$ & $50.5(44 ; 55)$ & $50(46 ; 52)$ & $0.288^{b}$ & $0.535^{b}$ \\
\hline AF burden [\%] & $36.9 \pm 44.5$ & $28.0 \pm 43.2$ & $18.3 \pm 37.5$ & $9.3 \pm 27.6$ & $0.027^{\mathrm{a}}$ & $0.162^{\mathrm{a}}$ \\
\hline Maximal HR during AF [bpm] & $143.3(146.5 ; 159.0)$ & $134(121 ; 142)$ & $129(112 ; 144)$ & $104(39.5 ; 137.5)$ & $0.088^{b}$ & $0.231^{b}$ \\
\hline Six-month follow-up - AF burden [\%] & $14.9 \pm 29.6$ & $1.7 \pm 6.3$ & $4.8 \pm 11.8$ & $7.9 \pm 26.6$ & $0.271^{\text {a }}$ & $0.366^{\mathrm{a}}$ \\
\hline LA diameter [mm] & $41.1 \pm 2,9$ & $39.1 \pm 4.2$ & $38.7 \pm 4.8$ & $37.9 \pm 3.5$ & $0.011^{\text {a }}$ & $0.135^{\mathrm{a}}$ \\
\hline LA volume index [mL/m²] & $28.6 \pm 10.2$ & $21.9 \pm 8.7$ & $26.7 \pm 12.0$ & $26.4 \pm 6.8$ & $0.565^{\mathrm{a}}$ & $0.333^{\mathrm{a}}$ \\
\hline LVEF [\%] & $58.5 \pm 5.0$ & $58.9 \pm 3.5$ & $57.6 \pm 4.7$ & $59.4 \pm 4.4$ & $0.675^{\text {a }}$ & $0.824^{a}$ \\
\hline Global four-chamber LA LS & $-9.4 \pm 4.2$ & $-10.1 \pm 4.4$ & $-13.0 \pm 3.6$ & $-13.5 \pm 5.7$ & $0.082^{\mathrm{a}}$ & $0.064^{\mathrm{a}}$ \\
\hline Global two-chamber LA LS & $-10.3 \pm 4.9$ & $-11.4 \pm 5.8$ & $-13.3 \pm 4.0$ & $-13.0 \pm 4.4$ & $0.156^{\mathrm{a}}$ & $0.242^{\mathrm{a}}$ \\
\hline$E / E^{\prime}$ & $8.7 \pm 4.4$ & $8.3 \pm 2.3$ & $7.7 \pm 2.4$ & $8.1 \pm 3.0$ & $0.565^{\mathrm{a}}$ & $0.853^{\mathrm{a}}$ \\
\hline Intima-media thickness [mm] & $0.73(0.63 ; 1.4)$ & $0.6(0.55 ; 1.3)$ & $0.6(0.5 ; 0.7)$ & $0.65(0.6 ; 0.9)$ & $0.894^{b}$ & $0.532^{b}$ \\
\hline
\end{tabular}

Data are shown as median and interquartile range or mean \pm standard deviation. Abbreviations - see Table 1

${ }^{*} 1^{\text {st }}$ vs. $4^{\text {th }}$ quartile - Student $t$ test $\left({ }^{(a)}\right.$ or Mann-Whitney $U$ test $\left({ }^{b}\right) ;{ }^{* *}$ ANOVA test $\left({ }^{a}\right)$ or Kruskal-Wallis test $\left({ }^{b}\right)$

kinase signalling pathway [26]. Li et al. [25] demonstrated that myocardial ischaemia-reperfusion injury led to lower myocardial renalase expression [25]. Renalase knock-out rats experienced greater extent of necrosis and apoptosis, which was prevented by pretreatment with recombinant renalase [25]. Consequently, less pronounced expression of renalase in patients with more advanced disease (e.g. greater LA diameter) may reflect impaired activation of protective mechanism. In addition, renalase was previously thought to be engaged in catecholamine metabolism and inversely correlated with peripheral and central blood pressure [20-22].

Our results support the notion that renalase depletion represents a marker of disease progression (Table 3) rather than a discriminator of $A F$ in the broad population because patients with AF had greater plasma renalase levels than the matched controls (Table 2). Undoubtedly, periprocedural plasma renalase did not herald recurrence of AF at six-month observation based on our findings (Fig. 3). Several other biomarkers were previously shown to predict maintenance of sinus rhythm. The largest GISSI-AF study by Latini et al. [16] performed on 382 patients with paroxysmal AF or after cardioversion for persistent AF provided evidence that high-sensitivity cardiac troponin $\mathrm{T}$, mid-range pro-A-type natriuretic peptide, $\mathrm{N}$-terminal pro-B-type natriuretic peptide, and $\mathrm{C}$-terminal pro-endothelin-1 were positively associated with AF recurrence. This confirms that well-established markers of myo- cardial necrosis (troponin) and atrial wall stress (natriuretic peptides) are better related with the long-term outcomes of rhythm control strategy [16]. In this study, a stable precursor of antidiuretic hormone, namely copeptin, failed to predict the relapse of AF [16].

In patients undergoing the PVI procedure, Takemoto et al. found that intracardiac galectin-3 concentration adequately identified patients at risk of AF recurrence following a single PVI [18]. Inhibition of this extracellular mediator of fibrosis in an animal model led to decreased AF burden and a tendency towards more rapid AF termination [18].

The results of the current study provided evidence that plasma renalase levels were paradoxically lower in the control group without the diagnosis of AF. Presumably, the prothrombotic and proinflammatory state of AF may upregulate the production of antiapoptotic renalase as a counter-regulatory mechanism [26]. Conversely, among individuals with confirmed AF, lower counter-regulatory response or depleted renalase spillover were associated with more advanced disease.

Taking into consideration the results of the study, circulating renalase seems to be an indicator of the stage of LA remodelling. Its concentration is strongly dependent on the systolic function of LA; global four-chamber LA longitudinal strain was the only independent predictor of plasma renalase concentration $(b=-0.425, p=0.0045)$. Conversely, renalase 


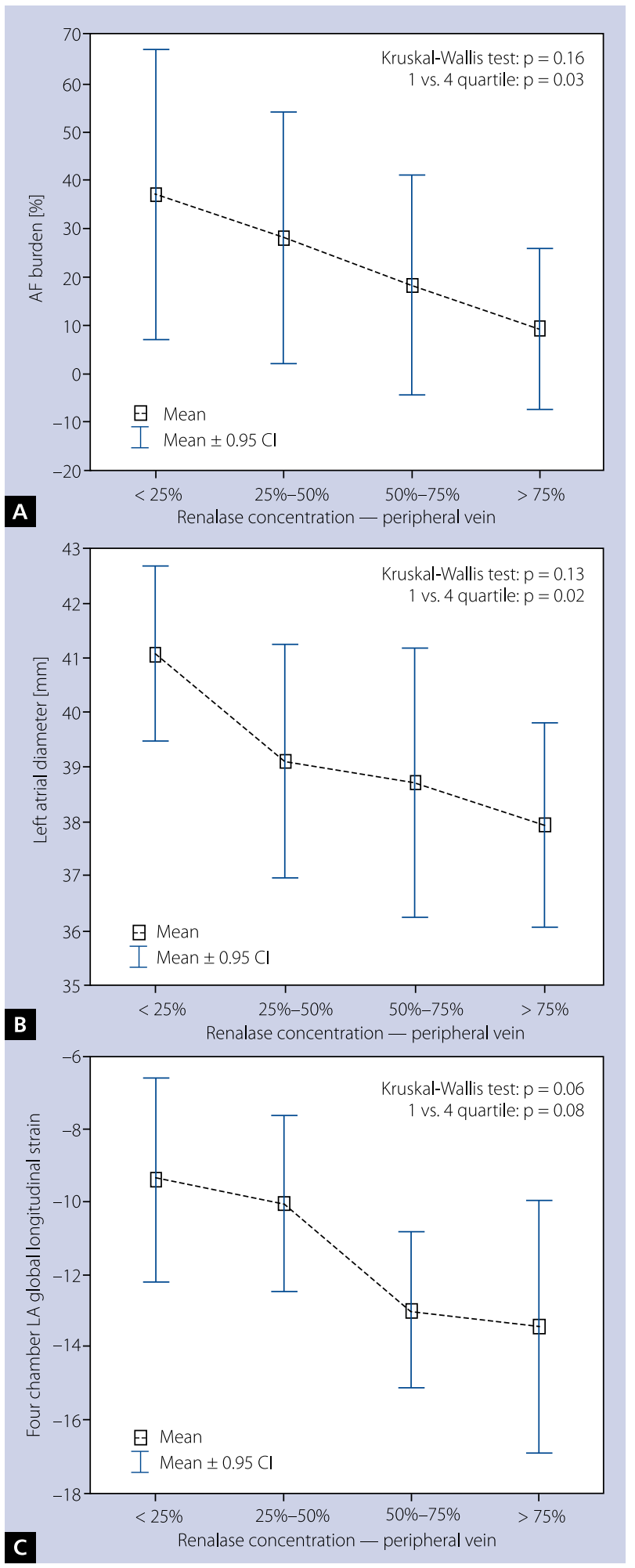

Figure 2. Atrial fibrillation (AF) burden, left atrial diameter, and four-chamber left atrial (LA) global longitudinal strain with respect to the quartile of peripheral vein renalase concentration; A. AF burden in relation to quartile of renalase concentration; B. LA diameter in relation to quartile of renalase concentration; C. Four-chamber LA global longitudinal strain in relation to quartile of renalase concentration; $\mathrm{Cl}$ - confidence interval

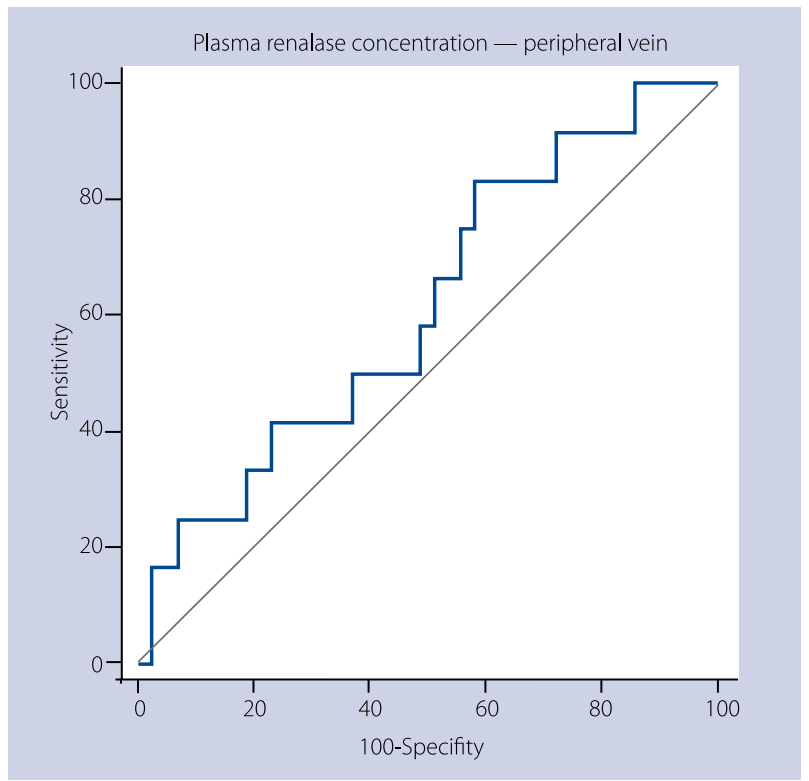

Figure 3. Receiver operating characteristic curve of the prediction of atrial fibrillation (AF) recurrence during six-month follow-up by periprocedural peripheral-vein renalase concentration

Table 5. Univariate regression analysis of different predictors of peripheral vein renalase concentration

\begin{tabular}{lcc} 
Renalase vs. & \multicolumn{2}{c}{ Renalase } \\
& - peripheral vein \\
\cline { 2 - 3 } & b & $\mathbf{p}$ \\
\hline Male sex & -0.02 & 0.899 \\
Age & 0.10 & 0.378 \\
Body mass index & -0.11 & 0.366 \\
CHA_DS - -VASc score & -0.01 & 0.933 \\
EHRA score & 0.23 & 0.057 \\
Arterial hypertension & -0.10 & 0.431 \\
Type 2 diabetes & -0.03 & 0.888 \\
Coronary artery disease & 0.11 & 0.375 \\
Hyperlipidaemia & 0.20 & 0.107 \\
In-hospital AF episodes & -0.21 & 0.134 \\
Maximal HR & -0.25 & 0.070 \\
Mean HR & -0.30 & 0.033 \\
AF burden & -0.32 & 0.023 \\
Paroxysmal AF & 0.20 & 0.104 \\
Maximal HR during AF episode & -0.42 & 0.050 \\
Mean platelet volume & 0.25 & 0.038 \\
Serum creatinine concentration & 0.10 & 0.415 \\
eGFR & -0.13 & 0.299 \\
Intima-media thickness & 0.03 & 0.874 \\
LVEF & 0.02 & 0.866 \\
E/E' & -0.07 & 0.620 \\
LA diameter & -0.29 & 0.019 \\
LA volume index & 0.02 & 0.880 \\
LV end-diastolic diameter & -0.25 & 0.051 \\
Global four-chamber LA LS & -0.36 & 0.008 \\
Global two-chamber LA LS & -0.27 & 0.062 \\
\hline
\end{tabular}

b - univariate regression coefficient; other abbreviations - see Table 1 
is not specific for AF, nor does it identify patients with a history of AF or reflect possible maintenance of sinus rhythm.

The main limitation of the study is a small study population. In particular, the analysis involved only seven patients with persistent $\mathrm{AF}$, which is characterised by more pronounced LA adverse remodelling. In addition, the authors did not investigate the temporal variability of plasma renalase concentration. The response to PVI was assessed by means of seven-day Holter electrocardiographic monitoring, which confers possible risk of underestimation of AF recurrence, as compared to e.g. home telemonitoring or implantable loop recorder.

In conclusion, low plasma renalase levels may be associated with impaired rate control, higher AF burden, and more advanced LA remodelling in patients with AF referred for PVI. Plasma renalase concentration failed to predict recurrence of $\mathrm{AF}$ in the six-month observation.

\section{Acknowledgements}

This work was supported by the institutional budget of the First Department of Cardiology, Medical University of Silesia, Katowice, Poland.

\section{Conflict of interest: none declared}

\section{References}

1. Kirchhof P, Benussi S, Kotecha D, et al. ESC Scientific Document Group. 2016 ESC Guidelines for the management of atrial fibrillation developed in collaboration with EACTS. Eur Heart J. 2016; 37(38): 2893-2962, doi: 10.1093/eurheartj/ehw210, indexed in Pubmed: 27567408.

2. Roy D, Talajic M, Nattel S, et al. Rhythm control versus rate control for atrial fibrillation and heart failure. N Engl J Med. 2008; 358(25): 2667-2677, doi: 10.1056/nejmoa0708789.

3. Van Gelder IC, Hagens VE, Bosker HA, et al. Rate Control versus Electrical Cardioversion for Persistent Atrial Fibrillation Study Group. A comparison of rate control and rhythm control in patients with recurrent persistent atrial fibrillation. $\mathrm{N}$ Engl J Med. 2002; 347(23): 1834-1840, doi: 10.1056/NEJMoa021375, indexed in Pubmed: 12466507.

4. Anter E, Callans DJ, Wyse DG. Pharmacological and electrical conversion of atrial fibrillation to sinus rhythm is worth the effort. Circulation. 2009; 120(14): 1436-1443, doi: 10.1161/CIRCULATIONAHA.108.824847, indexed in Pubmed: 19805660.

5. Ganesan AN, Shipp NJ, Brooks AG, et al. Long-term outcomes of catheter ablation of atrial fibrillation: a systematic review and meta-analysis. J Am Heart Assoc. 2013; 2(2): e004549, doi: 10.1161/JAHA.112.004549, indexed in Pubmed: 23537812.

6. Verma A, Jiang Cy, Betts TR, et al. STAR AF II Investigators. Approaches to catheter ablation for persistent atrial fibrillation. N Engl J Med. 2015; 372(19): 1812-1822, doi: 10.1056/NEJMoa1408288, indexed in Pubmed: 25946280.

7. Chao TF, Cheng CC, Lin WS, et al. Associations among the CHADS(2) score, atrial substrate properties, and outcome of catheter ablation in patients with paroxysmal atrial fibrillation. Heart Rhythm. 2011; 8(8): 1155-1159, doi: 10.1016/j. hrthm.2011.03.016, indexed in Pubmed: 21402172.

8. Hung CY, Lin CH, Wang KY, et al. Dosage of statin, cardiovascular comorbidities, and risk of atrial fibrillation: a nationwide population-based cohort study. Int J Cardiol. 2013; 168(2): 1131-1136, doi: 10.1016/j.ijcard.2012.11.087, indexed in Pubmed: 23218193.
9. Wang T, Wang M, Fung JWH, et al. Atrial strain rate echocardiography can predict success or failure of cardioversion for atrial fibrillation: a combined transthoracic tissue Doppler and transoesophageal imaging study. Int J Cardiol. 2007; 114(2): 202-209, doi: 10.1016/j.ijcard.2006.01.051, indexed in Pubmed: 16822565.

10. Nakanishi K, Fukuda S, Yamashita H, et al. High-sensitive cardiac troponin $\mathrm{T}$ as a novel predictor for recurrence of atrial fibrillation after radiofrequency catheter ablation. Europace. 2017; 19(12): 1951-1957, doi: 10.1093/europace/euw314, indexed in Pubmed: 28069837.

11. Benito EM, Carlosena-Remirez A, Guasch E, et al. Left atrial fibrosis quantification by late gadolinium-enhanced magnetic resonance: a new method to standardize the thresholds for reproducibility. Europace. 2017; 19(8): 1272-1279, doi: 10.1093/europace/euw219, indexed in Pubmed: 27940935.

12. Suksaranjit $P$, Akoum N, Kholmovski EG, et al. Incidental LV LGE on CMR Imaging in Atrial Fibrillation Predicts Recurrence After Ablation Therapy. JACC Cardiovasc Imaging. 2015; 8(7): 793-800, doi: 10.1016/j.jcmg.2015.03.008, indexed in Pubmed: 26093929.

13. Di Salvo G, Caso P, Lo Piccolo R, et al. Atrial myocardial deformation properties predict maintenance of sinus rhythm after external cardioversion of recent-onset lone atrial fibrillation: a color Doppler myocardial imaging and transthoracic and transesophageal echocardiographic study. Circulation. 2005; 112(3): 387-395, doi: 10.1161/CIRCULATIONAHA.104.463125, indexed in Pubmed: 16006491.

14. Melduni RM, Lee HC, Bailey KR, et al. Real-time physiologic biomarker for prediction of atrial fibrillation recurrence, stroke, and mortality after electrical cardioversion: A prospective observational study. Am Heart J. 2015; 170(5): 914-922, doi: 10.1016/j. ahj.2015.07.027, indexed in Pubmed: 26542499.

15. Degener S, Pattberg SV, Feuersenger H, et al. Predictive value of B-type natriuretic peptide levels in patients with paroxysmal and persistent atrial fibrillation undergoing pulmonary vein isolation. J Interv Card Electrophysiol. 2011; 30(3): 217-225, doi: 10.1007/s10840-010-9540-2, indexed in Pubmed: 21249437.

16. Latini R, Masson S, Pirelli S, et al. GISSI-AF Investigators. Circulating cardiovascular biomarkers in recurrent atrial fibrillation: data from the GISSI-atrial fibrillation trial. J Intern Med. 2011; 269(2): 160-171, doi: 10.1111/j.1365-2796.2010.02287.x, indexed in Pubmed: 20964739.

17. Henningsen KM, Nilsson B, Bruunsgaard H, et al. Prognostic impact of hs-CRP and IL-6 in patients undergoing radiofrequency catheter ablation for atrial fibrillation. Scand Cardiovasc J. 2009; 43(5): 285-291, doi: 10.1080/14017430802653676, indexed in Pubmed: 19117239.

18. Takemoto Y, Ramirez RJ, Yokokawa M, et al. Galectin-3 regulates atrial fibrillation remodeling and predicts catheter ablation outcomes. JACC Basic Transl Sci. 2016; 1(3): 143-154, doi: 10.1016/j. jacbts.2016.03.003, indexed in Pubmed: 27525318.

19. Nakazawa Y, Ashihara T, Tsutamoto T, et al. Endothelin-1 as a predictor of atrial fibrillation recurrence after pulmonary vein isolation. Heart Rhythm. 2009; 6(6): 725-730, doi: 10.1016/j. hrthm.2009.02.027, indexed in Pubmed: 19467500.

20. $\mathrm{Xu} \mathrm{J,} \mathrm{Li} \mathrm{G,} \mathrm{Wang} \mathrm{P,} \mathrm{et} \mathrm{al.} \mathrm{Renalase} \mathrm{is} \mathrm{a} \mathrm{novel,} \mathrm{soluble} \mathrm{monoamine}$ oxidase that regulates cardiac function and blood pressure. J Clin Invest. 2005; 115(5): 1275-1280, doi: 10.1172/JCI24066, indexed in Pubmed: 15841207.

21. Desir GV. Renalase is a novel renal hormone that regulates cardiovascular function. J Am Soc Hypertens. 2007; 1(2): 99-103, doi: 10.1016/j.jash.2006.12.001, indexed in Pubmed: 20409839.

22. Wybraniec MT, Mizia-Stec K, Trojnarska O, et al. Low plasma renalase concentration in hypertensive patients after surgical repair of coarctation of aorta. J Am Soc Hypertens. 2014; 
8(7): 464-474, doi: 10.1016/j.jash.2014.04.009, indexed in Pubmed: 25064768.

23. Zhao Qi, Fan Z, He J, et al. Renalase gene is a novel susceptibility gene for essential hypertension: a two-stage association study in northern Han Chinese population. J Mol Med (Berl). 2007; 85(8): 877-885, doi: 10.1007/s00109-006-0151-4, indexed in Pubmed: 17216203 .

24. Farzaneh-Far R, Desir GV, Na B, et al. A functional polymorphism in renalase (Glu37Asp) is associated with cardiac hypertrophy, dysfunction, and ischemia: data from the heart and soul study. PLoS One. 2010; 5(10): e13496, doi: 10.1371/journal. pone.0013496, indexed in Pubmed: 20975995.

25. Li X, Xie Z, Lin M, et al. Renalase protects the cardiomyocytes of Sprague-Dawley rats against ischemia and reperfusion injury by reducing myocardial cell necrosis and apoptosis. Kidney Blood Press Res. 2015; 40(3): 215-222, doi: 10.1159/000368497, indexed in Pubmed: 25966766.

26. Wang L, Velazquez H, Chang J, et al. Identification of a receptor for extracellular renalase. PLoS One. 2015; 10(4): e0122932, doi: 10.1371/journal.pone.0122932, indexed in Pubmed: 25906147.

Cite this article as: Wybraniec MT, Wieczorek J, Woźniak-Skowerska I, et al. Renalase is associated with adverse left atrial remodelling and disease burden in patients with atrial fibrillation undergoing pulmonary vein isolation. Kardiol Pol. 2018; 76(8): 1232-1241, doi: 10.5603/KP.a2018.0108.

\section{WHAT IS NEW?}

There is a paucity of data concerning reliable laboratory predictors of response to ablation of atrial fibrillation. Renalase is a kidney-derived protein that is related to turnover of plasma catecholamines. In this study we found that low plasma renalase concentration is associated with impaired rate control, higher atrial fibrillation burden, and more advanced left atrial remodelling. Plasma renalase did not accurately predict sinus rhythm maintenance at six-month follow-up. 